\title{
Representaciones mentales y sociales en la equidad de género
}

\author{
Ana María Winfield Reyes ${ }^{1}$, \\ Yasmín Ivette Jiménez Galán² y \\ Carlos Topete Barrera ${ }^{3}$
}

\footnotetext{
${ }^{1}$ Instituto Politécnico Nacional, Ciudad de México, México. Correo electrónico: amwr76@gmail.com

${ }^{2}$ Instituto Politécnico Nacional, Ciudad de México, México. Correo electrónico: yjimenezg@ipn.mx

${ }^{3}$ Instituto Politécnico Nacional, Ciudad de México, México. Correo electrónico: cartopba@yahoo.com
}

\section{Resumen}

La equidad de género implica la igualdad de oportunidades y condiciones para todo ser humano, para su pleno desarrollo; responde a los derechos universales, a la justicia como cualidad que mueve a dar a cada uno lo que merece y que permite la construcción de sociedades observadoras de la dignidad humana. Si bien la equidad de género asume una relación de justicia respecto al otro, ésta se ve afectada por los constructos sociales, las representaciones mentales y las formas de ejercer el poder de acuerdo a los usos y costumbres de cada cultura, de manera que dicha equidad difícilmente se cumple en lo cotidiano e históricamente se vulnera a los grupos minoritarios o en desventaja. A nivel institucional es posible fomentar la equidad de género y con ello una cultura de valores que reditúe en mayor ciudadanía. Por lo tanto es necesario identificar los constructos que tienen los sujetos y que los ligan a determinadas prácticas, de ahí que el objetivo de esta investigación fue explorar las representaciones mentales que conforman la equidad de género manifestadas a través del lenguaje de estudiantes de educación superior. Con el análisis cualitativo de la in- 
formación se generaron 131 códigos que nutrieron una base de datos para un sistema computarizado que pretende ser herramienta para analizar datos de nuevos informantes. Se usó la herramienta metodológica del Atlas.ti para generar un mapa conceptual interpretativo acerca de las representaciones mentales de la equidad de género y esto permitió el andamiaje de propuestas de acción a partir de la comprensión de dichas representaciones.

Palabras clave: equidad de género, ciudadanía, representaciones mentales.

\begin{abstract}
Gender equality implies equal opportunities and conditions for every human being, for them to achieve their full development. Gender equality responds to universal rights and justice, which is a quality that fuels the individual to give everyone what they deserve, and allows the construction of societies that are on the look for human dignity. Although gender equality takes over a relation of justice with others, this relation is affected by social constructs, mental representations, and different ways of exercising power adhering to the ways and customs of each culture, thus equity hardly emerges in the daily life, and historically has violated minority or disadvantaged groups. At an institutional level, it is possible to promote gender equality and later, a culture of values that bears greater civic responsibility through specific actions. To fulfill these proposals, the subject's constructs and mental representations that bind the individual to certain practices must be identified; hence the objec-
\end{abstract}


tive of this research was to explore the mental representations that make gender equality expressed through language of students in higher education. With the qualitative analysis of the information 131 codes were generated which nurtured a data base for a computer system that serves as a tool to analyze new information. Atlas.ti was the software used to create the interpretative conceptual network about mental representation of the equity of genre and these allowed the scaffolding of proposals for action from the understanding that these representations generated.

Keywords: gender equality, civic responsibility, mental representations.

RECEPCIÓN: 6 DE ABRIL DE 2016 / ACEPTACIÓN: 24 DE MAYO DE 2016

\section{Introducción}

Este estudio explora, desde la perspectiva de género, las representaciones mentales alusivas a la equidad de género en estudiantes de educación superior a partir del análisis de frases incompletas y a la luz del abordaje de la teoría fundamentada como herramienta de análisis que revisa y contrasta los constructos teóricos que se han hecho respecto a la equidad de género, la formación ciudadana y las representaciones mentales con los datos obtenidos del trabajo de campo. En el caso de este trabajo, se parte de considerar a la Institución de Educación Superior el instrumento por el cual los 
estudiantes articulan y, en un momento dado, modifican sus estructuras y representaciones mentales mediante las experiencias y espacios que permiten la discusión de temáticas éticas como son la equidad de género y la formación ciudadana.

\section{Impacto de la institución educativa en las} representaciones mentales como forjadora de ciudadanía

La equidad consiste en permear las posibilidades a distintos grupos que históricamente han sido vulnerados (el de las mujeres por ejemplo), pero este discurso se enfrenta a los desafíos de la realidad misma en la que confluyen diferentes fuerzas y contradicciones, donde por una parte se reconoce con mayor énfasis la importancia del fomento de una cultura de valores en que la justicia se manifieste dando a cada uno lo que corresponde, y por otra, se dan prácticas arraigadas en las representaciones mentales de la cultura y la época a la que se pertenece y que contradicen esa intención de justicia social.

Muchas políticas e iniciativas a nivel nacional e internacional destacan la importancia de avanzar hacia la igualdad, ya que ésta proveerá y será signo de sociedades más justas, donde la formación ciudadana y compromiso estarán presentes. Sin embargo, al analizar de manera más profunda las prácticas inherentes a las relaciones de género encontramos que: 
el eje del problema ha sido el concepto de desigualdad, que implica la idea de que la diferencia sexual siempre ha sido socialmente valorada en términos jerárquicos que señalan negativamente a lo femenino y positivamente a lo masculino. Esto se ha expresado históricamente como la subordinación social de las mujeres al poder masculino, donde encontramos como presencia constante una relación de dominación unívoca (Serret, Alfie y García, 1991: 422).

En el caso de México, por ejemplo, a pesar de que los derechos fundamentales erogados por la Constitución Mexicana reconocen la igualdad del hombre y de la mujer y la no discriminación, las prácticas de la sociedad mexicana tienden más a lo tradicional; es decir: la persona que ostenta el poder o domina el espacio público, generalmente ha sido el hombre, en tanto ha quedado reservado el espacio privado para la mujer. Esto ha generado a lo largo de décadas que las mujeres y las niñas (aunque no son los únicos grupos afectados) se vean limitadas en sus posibilidades de elección y decisión en temas relacionados con la carrera profesional, la familia, la distribución del gasto familiar, etc.

Derivado de lo anterior, el gobierno Federal ha impulsado iniciativas hacia la igualdad entre mujeres y hombres que quedan plasmadas en la ley del 2001 al crearse el Instituto Nacional de las Mujeres y más tarde, en 2006, la Ley General para la Igualdad de Hombres y Mujeres y el Programa Nacional para la Igualdad de Oportunidades y la no Discriminación de las Mujeres, donde se 
hace transversal la perspectiva de género en los tres poderes de gobierno, creándose en 2013 el comité del CEAMEG o Centro de Estudios para el Adelanto de las Mujeres y la Equidad de Género.

Por lo tanto, la igualdad de género en México ha sido la meta u objetivo de las políticas públicas, pero para lograrla se debe partir de una equidad de género que reconozca las diferencias existentes en distintos grupos de la sociedad mexicana; con la equidad se pretenden crear las condiciones para que dichas diferencias no impidan a las personas el acceso a las mismas oportunidades de desarrollo personal, económico y político.

Así, la falta de equidad de género se formula como una problematización de la desigualdad socialmente producida entre los sexos que aleja determinantemente la justicia social tan buscada aunque funja como un aparente esquema simbólico ordenador de la cultura, donde sus procesos juegan un papel en la constitución de las subjetividades y de la cultura (Serret, Alfie y García, 1991).

Es importante resaltar que de acuerdo con la Organización de las Naciones Unidas para la Agricultura y la Alimentación (FAO) (2011) la esencia de la equidad no radica en el tratamiento idéntico a los diferentes grupos, ya que éste puede ser igual o diferente pero siempre deberá considerarse equivalente en términos de derechos, beneficios, obligaciones y oportunidades.

La idea de equidad hace énfasis en el rescate del ser humano en tanto sus posibilidades, de ahí que se pretenda cambiar desde una perspectiva de género - enfoque en la investigación que permite hacer evidentes las diferencias atribuidas socialmente a hom- 
bres y mujeres- y desde la acción conjunta de las instituciones, el rumbo de supuesta neutralidad universal de los individuos sin diferencias ni privilegios por el reconocimiento de que, aunque diferentes, las mujeres y los hombres tienen igualdad de derechos, dejando atrás la lucha de géneros que desconoce o asume papeles del otro y promoviendo así un cambio cultural donde los hombres y las mujeres se reconozcan, respeten y se conciban como pares en medio de las transformaciones progresivas (PROIGUALDAD, 2013; Buitrago, Cabrera y Guevara, 2009).

Dentro de este contexto, es labor de la organización educativa favorecer los espacios en que esta apremiante cultura de valores se haga realidad más allá del discurso, es decir, en la práctica misma. Proyecto ambicioso que inicia a partir de la comprensión de lo que mueve a los individuos cuando se tratan temas de equidad de género, formación ética y ciudadana; tópicos abordados en este estudio desde la comprensión de las representaciones mentales.

En un mundo globalizado con marcadas diferencias culturales, sociales, políticas y económicas, la sociedad exige a las Instituciones de Educación Superior que den respuesta y cumplan la función de formación de ciudadanos que potencien las instituciones democráticas, la equidad de género y otras funciones instrumentales ligadas al mundo de la economía (Dewey, 1998; Giddens, 2000; Bordieu, 2008; González, 2010).

Entonces, se concibe a la educación como el derecho inalienable de los individuos que a través de sus instituciones permite al Estado transmitir las concepciones y los valores que serán capaces 
de cambiar a la sociedad en su conjunto. No obstante Bernstein (1993), Habermas (2007) y Bordieu (2008) han insistido en observar el carácter reproductor de las estructuras sociales existentes donde a través del lenguaje y el discurso manifiesto, las instituciones y, en este caso la escuela, legitiman los paradigmas sociales dominantes (González, 2010).

Por otro lado, la igualdad ante la ley es la base del concepto de ciudadanía y la formación en ciudadanía permite dotar a los individuos de las competencias necesarias para participar y vivir en democracia, en el mundo contemporáneo de las grandes urbes, que no se limita a adquirir derechos y obligaciones por parte del Estado sino que requiere que los individuos desarrollen la cualidad moral de pertenecer a una sociedad para asumir esos derechos y obligaciones, donde educación y democracia son indisolubles (González, 2010; Guevara, 1998).

Asimimo, para Latapí (1993), la tan buscada calidad educativa tiene como componentes esenciales la distribución equitativa de la educación, el desarrollo de la conciencia ciudadana y del sentido de la responsabilidad social, configurando al Estado como el responsable de la misma.

Entonces, la concepción de la equidad de género se legitima desde la articulación de las políticas públicas en la institución educativa y se manifiesta a través del lenguaje de sus actores, lenguaje que da cuenta de la integración de contenidos simbólicos y sociales en las representaciones mentales. 
Por ser las Instituciones de Educación Superior el lugar donde el individuo asimila la cultura de la sociedad a la que pertenece, se considera que es en la escuela donde los estudiantes podrían configurar una nueva cultura de colaboración, respeto y compromiso, debido a que es ahí donde se busca un conocimiento científico frente al saber vulgar, se fomenta la actitud crítica frente a la asimilación ingenua del orden establecido y se logra el desarrollo moral de los individuos y de los pueblos a través del fomento de la ética (Santos, 1996). Esto a partir de hacer manifiestos los contenidos simbólicos que emergen de algún tópico dirigido.

De ahí que en la intención de dar cabida a la comprensión de los fenómenos que subyacen a las prácticas institucionales éticas, la formación ciudadana, la justicia como un valor vivencial, la equidad de género y el estudio de las representaciones mentales y sociales surge como una posibilidad certera de acercarse a las concepciones que mueven la cultura institucional y la vida simbólica de los actores de esa institución y permite transformar la realidad en tanto se acerca a ella.

El abordaje de la equidad de género, desde las representaciones mentales, nace del supuesto en el que éstas son sistemas cognoscitivos con lógica y lenguaje propio que dirigen no solamente la apreciación que se tiene de las cosas, sino también la conducta.

La representación mental es una forma específica del conocimiento cuya función responde a la elaboración del comportamiento y la comunicación entre los individuos, es decir, es el corpus organizado de conocimientos mediante el cual los sujetos interpre- 
tan la realidad que viven mediada por los valores éticos y morales, las necesidades, los roles sociales y otros aspectos socio culturales (Moscovici en Mora, 2002; Materán, 2008).

Así entonces, las representaciones sociales son la visión o pensamiento que los individuos o grupos tienen y utilizan para comprender el mundo y elaborar información sobre los objetos de la realidad cotidiana, con un origen social, pues son la interiorización de experiencias, modelos, conductas y pensamientos socialmente transmitidos, y por tanto, resultan esenciales para comprender la dinámica de la interacción y de las prácticas sociales relacionadas en este caso con el género, entendido como un elemento constitutivo de las relaciones sociales basadas en las diferencias que distinguen los sexos y que está fuertemente imbricado en las jerarquías sociales constituyendo una forma a través de la cual se estructuran las relaciones de poder entre las personas (Jimeno en Buitrago, Cabrera y Guevara, 2009).

La representación social es un sistema inserto dentro de otro más amplio que es el sistema cultural. Las representaciones ayudan a dotar de sentido la existencia de los individuos estructurando una distinción social, un orden y formas invisibles de poder (Camberos, 2011:46).

Por lo tanto, las representaciones cumplen las funciones de hacer convencionales a los sujetos, objetos y eventos cotidianos (convertir lo extraño en familiar), propiciar la comunicación interpersonal, promover el pensamiento colectivo sobre lo correcto e incorrecto dentro de la sociedad a la que se pertenece y justificar las decisiones y acciones de las personas (Materán, 2008). 
En concordancia con Bordieu, se hace necesario entender cómo los y las jóvenes se ven actualmente, en tanto hombres y mujeres, cómo perciben a los demás y cómo sus relaciones se ven afectadas por estas percepciones, en tanto sujetos sexuados que ya han asimilado e interiorizado el discurso sobre lo que culturalmente se considera propio de un determinado sexo (Buitrago, Cabrera y Guevara, 2009).

\section{Metodología de las representaciones mentales y sociales en la equidad de género: un estudio exploratorio cualitativo}

Desde una metodología cualitativa, el presente estudio exploró las representaciones mentales que tienen estudiantes de nivel superior en relación a la equidad de género, así como las prácticas asociadas a este concepto, a fin de proponer líneas de acción institucionales que favorezcan la equidad de género y la formación ciudadana.

Los objetivos específicos que se plantearon desde un principio para lograr la meta final fueron los siguientes:

1. Identificar los elementos principales del
constructo de equidad de género en la comunidad estu-
diantil, así como las prácticas inherentes a dicho
constructo.

2. Elaborar un sistema que permita el manejo y procesamiento de los datos observados. 
3. Generar una propuesta que fomente la formación de estudiantes con una cultura cuyos pilares sean la ciudadanía, la equidad de género y la justicia social.

La primera de las fases de investigación incluyó la revisión del estado del arte acerca de la equidad de género, las representaciones mentales y la formación ciudadana. A partir de esta revisión teórica y desde la teoría fundamentada se construyeron tres categorías de observación, las cuales fueron: representaciones mentales en torno a la mujer, representaciones mentales en torno al hombre y representaciones vinculadas a la identidad. De cada categoría se idearon frases incompletas que dieran cuenta de los constructos relacionados a esas representaciones, mismas que se pidió a los sujetos contestaran con lo primero que se les viniera a la mente. Algunos ejemplos se presentan a continuación:

\section{Representaciones mentales en torno a la mujer}

1. Las mujeres adultas...

2. La mujer desde niña es educada para...

3. Se espera que las mujeres...

4. Las jóvenes de hoy...

5. Hoy día se ve a la mujer como...

6. Ser mujer significa...

7. Lo que más rechazo de las mujeres es... 


\section{Representaciones mentales en torno al hombre}

8. El hombre se encarga de...

9. El hombre desde niño es educado para...

10. Se espera que los hombres...

11. Lo que más rechazo de los hombres...

\section{Representaciones vinculadas a la identidad de género}

12. Los retos que tengo son...

13. Lo que más aprecio de ser hombre/mujer...

14. Como hombre / mujer, me preocupa que se me considere...

En una segunda fase se aplicó el instrumento a seis grupos (cuatro del primer nivel de la carrera de Ingeniería en Sistemas y dos del segundo nivel de la misma carrera), donde se obtuvieron 178 registros. Las características de los sujetos de investigación, en cuanto a la conformación de sus grupos, fueron que la población femenina en cada uno de ellos no superaba los cuatro integrantes, las edades iban de los 19 a los 23 años y todos ellos eran alumnos regulares de ingeniería. En la tabla 1 se muestran algunas de las respuestas que los sujetos escribieron.

Cada registro contuvo la respuesta a las catorce frases incompletas y estas frases fueron, en una tercera fase de investigación, analizadas y codificadas por los investigadores mediante el procedimiento de triangulación de la interpretación apoyándose de la herramienta metodológica del Atlas.ti, generando 131 códigos presentes en las tres grandes categorías mencionadas anteriormente (ver Tabla 2). 
Tabla 1. Ejemplo de respuestas a frases incompletas

\begin{tabular}{|c|c|c|}
\hline 5. Se espera que las mujeres & 7. Los jóvenes de hoy & 8. Hoy día se ve a la mujer como \\
\hline $\begin{array}{l}\text { Sean emprendedoras y no } \\
\text { dependientas de los hombres }\end{array}$ & $\begin{array}{l}\text { No se desarrollan completa- } \\
\text { mente como mujer }\end{array}$ & $\begin{array}{l}\text { Una persona muy productiva } \\
\text { para la sociedad }\end{array}$ \\
\hline $\begin{array}{l}\text { Toman el mismo papel } \\
\text { que el hombre }\end{array}$ & Son el futuro del mañana & Como un ser humano normal \\
\hline Sean comprensivas & No les importa la cultura general & $\begin{array}{l}\text { No me gusta compararlos son mujeres } \\
\text { y para mí eso es maravilloso }\end{array}$ \\
\hline Sobresalgan & $\begin{array}{l}\text { Están cambiando de } \\
\text { mentalidad rápidamente }\end{array}$ & Una fuerza en potencia \\
\hline Consigan la equidad de género & $\begin{array}{l}\text { Son inmaduras y no saben lo que } \\
\text { quieren }\end{array}$ & $\begin{array}{l}\text { Una persona con las mismas } \\
\text { capacidades que los demás }\end{array}$ \\
\hline Sean líderes & Buscan el dinero & Objeto \\
\hline Sobresalgan con más dificultad & Son más alocadas que los hombres & Un ser con derechos \\
\hline Tomen 2 roles & $\begin{array}{l}\text { Hacen lo que quieren por que } \\
\text { se les hace fácil }\end{array}$ & El sexo dominante \\
\hline Sean fuertes & Se preparan profesionalmente & Una persona \\
\hline Seamos lo que queremos ser & $\begin{array}{l}\text { Son diferentes, no me gusta } \\
\text { generalizar porque no me siento } \\
\text { o nos siento, iguales en ese } \\
\text { aspecto }\end{array}$ & Un ser fuerte y capaz \\
\hline Ser mantenida & $\begin{array}{l}\text { No se hacen responsables } \\
\text { de sus errores }\end{array}$ & El sexo dominante \\
\hline Alcancen sus metas en la vida & Son muy locas y locos & Objeto sexual, aunque se trate de valorar \\
\hline Hagan el quehacer & Son el futuro de nuestra nación & Una persona libre y feliz \\
\hline Tengan los mismos derechos & Quieren hacer cosas más abiertas & Una persona que podría sobresalir a otros \\
\hline Tengan liderazgo & Buscan igualdad y superación & Trabajadora \\
\hline
\end{tabular}

Fuente: elaboración personal. 
Tabla 2. Listado de códigos generados a través del análisis cualitativo de la información

\begin{tabular}{|c|c|c|c|}
\hline LAbur & 34. Mrimulited & 6. Hramensa & ID. Parsmis \\
\hline 2.At+audud & 15. Dumbrasuch & Ba Iroudad & LLL. Farmidad \\
\hline 3. Anuthlud & 3. Dhwabrom & D. Lnpeterat & IDI. Pramins \\
\hline 4. Anblis & Bo Difulad & Do humie & 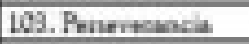 \\
\hline 5. Ampily & 3 Deredt & Th. Trkipacits & 14. Pemmith \\
\hline Ant An & D Dirats & 11 Inderrderein & LOS Prakiss \\
\hline 7. Andourin & 4. Dhecrimiraciba & 13indiversin & Ith Praperain \\
\hline An Ariounda & 4L. Dhernats & 7t Inturiended & LA7, Pramastits \\
\hline Fingria & Al Edmorit- & Ih Inicivint & 14. Promeribs \\
\hline I0. Apora & 41. Escling & 7D. honduse. & IVI. Ronted \\
\hline IL Aprais & 44. Irising & 7. Thumedud & IIA. Rruliurdo \\
\hline II Amotris & 45. Entrudeinimso & D. Inupineus & ILL Rerta \\
\hline 13 Aravi & 4h. Evadud & 79. In:ardad & 1L2. Reipenubludud \\
\hline It humreids & 47. Exhutso & Di Invilyonas & LII, Finde: \\
\hline D. Ausungirls & 4 Equihal & S. Inwhrenis & 1H. Riquan \\
\hline 10. Erpotscidud & 11. Entuntipe: & Bt Irowpornthided & L1, R-un \\
\hline 17. Eand at & 91 Engra & Dh LAmis & Lbistiris \\
\hline It Filla & SLEure & St. Lh+m & 117. Strardud \\
\hline I0. Bupudal & 51 Eypirmin & B. Litumad & ILI Straden \\
\hline 10.hudadals & 5i. Furila & S6. LH+masta & LLA Stw: \\
\hline 1LEbudh & SHFth & Bi. Mothes & 1X. Secil \\
\hline M Constivited & 5 Folisale & MS Mare: & 17L Son ain th \\
\hline A Emmanibn & 5. Furime & EA Mrive & 172 Sopest \\
\hline it corima & Iit Flimen & 50 Mr-ipulacte & 173, Surnifis \\
\hline HEstiming & 4. Foridhot & 91. Mvimitura & 134. Suprocke \\
\hline 3 Erituitan & If Fruser & 91. Musmits & 175. Supmaind \\
\hline P.Asmal & di. Foma & 91. Mhusdrin & 18d. Troudeta \\
\hline 8. Cownmpin & dLCrningbia & 9. Na coming: & 172. Thamendur \\
\hline MLminis & b2 Emong & Ha Chthmo & 12. Hula \\
\hline Mcru & 6) Hey & Ge ctus & 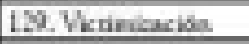 \\
\hline 3L Thinded & dH. Harnotiden & 97. Gporminden & L9. Yilareis \\
\hline 1: Deperdiniu & Di Handed & Fon Drulk & LIL Yosm \\
\hline 33. Thucrifina & Di. Herids & 59. Brifindids & \\
\hline
\end{tabular}

La codificación generada a partir del análisis cualitativo de la información permitió nutrir una base de datos para un sistema computarizado que pretende seguir indagando en línea la información respecto a las representaciones mentales de equidad de género de estudiantes y seguir integrando la comprensión de los constructos mentales y sociales que subyacen a la equidad de género (ver Figura 1). 
Figura 1. Interfaz del sistema de evaluación institucional de la equidad de género y despliegues de frases incompletas

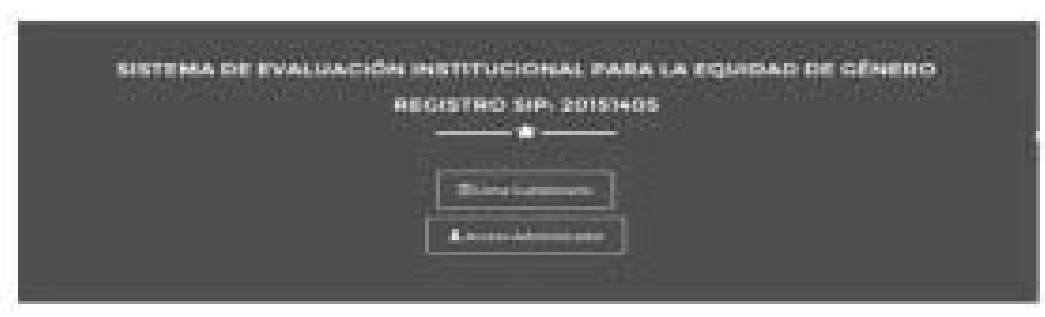

Evaluar Cuestionarios
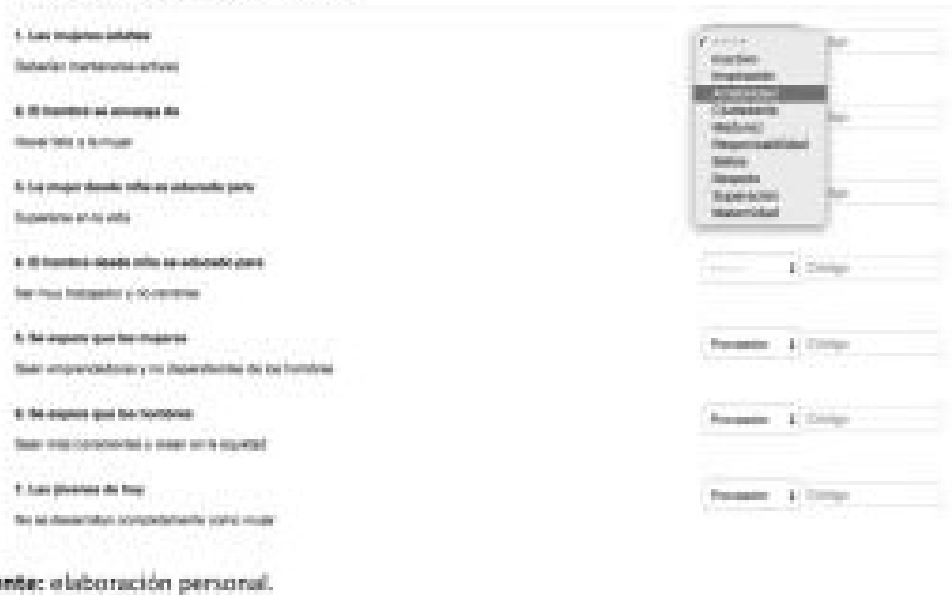

Tuente: abibaración pernanul.

Este sistema permite procesar de manera especializada la información vertida en él, generando un reporte preliminar (ver Figura 2).

\section{Resultados de la exploración de las representaciones} mentales en la equidad de género

A continuación se presenta la información que destacó en el análisis cualitativo de la información; retomando tres de los códigos que aparecieron con mayor iteración en cada frase incompleta. Esos códigos están asociados a las frases escritas por los informantes y se 
Figura 2. Despliegue de reporte del sistema de evaluación de equidad de género

Preguntas: Las mujeres adultas

\begin{tabular}{lc}
\hline Palabras & Iteraciones \\
\hline Inactivo & 1 \\
\hline Inspiración & 1 \\
\hline Amabilidad & 4 \\
\hline No contestó & 11 \\
\hline Son ejemplo & 1 \\
\hline Ciudadana & 1 \\
\hline Madurez & 8 \\
\hline Responsabilidad & 9 \\
\hline Independencia & 6 \\
\hline Bellas & 1 \\
\hline Independencia & 2 \\
\hline Sabiduría & 12 \\
\hline Inmadurez & 2 \\
\hline Respeto & 2 \\
\hline Superación & 1 \\
\hline Maternidad & 3 \\
\hline Igualdad & 3 \\
\hline Gerontofobia & 1 \\
\hline Experiencia & 4 \\
\hline Fuente: & \\
\hline
\end{tabular}

Fuente: elaboración personal. 
muestran ya clasificados por las categorías de análisis arriba mencionadas (ver tablas 3, 4 y 5).

En las tablas 6, 7 y 8 se presenta la codificación desprendida de cada pregunta dentro de las categorías de análisis y en la figura 3 un mapa conceptual interpretativo con ayuda de la herramienta metodológica computarizada del Atlas.ti.

Tabla 3. Representaciones mentales en torno a la mujer

\begin{tabular}{llc}
\hline Frase incompleta & Código asociado a respuestas & Iteración \\
\hline Las mujeres adultas... & Sabiduría & 12 \\
\hline La mujer desde niña es educada para... & Hogar & 24 \\
\hline Se espera que las mujeres... & Hogar & 10 \\
\hline Las jóvenes de hoy... & Inmadurez & 22 \\
\hline Hoy día se ve a la mujer como... & Igualdad/Equidad & 24 \\
\hline Ser mujer significa... & No contestó & 23 \\
\hline Lo que más rechazo de las mujeres es... & No contestó & 17 \\
\hline
\end{tabular}

Fuente: elaboración personal.

Tabla 4. Representaciones mentales en torno al hombre

\begin{tabular}{llc}
\hline Frase incompleta & Código asociado a respuestas & Iteración \\
\hline El hombre se encarga de... & Laboral/Proveedor & 43 \\
\hline El hombre desde niño es educado para... & Laboral/Proveedor & 27 \\
\hline Se espera que los hombres... & Laboral & 12 \\
\hline Lo que más rechazo de los hombres... & Machismo & 31 \\
\hline
\end{tabular}

Fuente: elaboración personal. 
Tabla 5. Representaciones vinculadas a la identidad de género

\begin{tabular}{llc}
\hline Frase incompleta & Código asociado a respuestas & Iteración \\
\hline Los retos que tengo son... & Estudios & 29 \\
\hline Lo que más aprecio de ser & $\begin{array}{l}\text { No contestó } \\
\text { hombre/mujer... }\end{array}$ & $\begin{array}{l}\text { Fortaleza } \\
\text { (contestado por mujeres) } \\
\text { Libertad } \\
\text { (contestado por hombres) }\end{array}$ \\
\hline Como hombre/mujer, me preocupa & $\begin{array}{l}\text { Machismo } \\
\text { (respuesta de hombres) } \\
\text { que se me considere... }\end{array}$ & $\begin{array}{l}\text { Incapacidad } \\
\text { (respuesta de mujeres) }\end{array}$ \\
\hline
\end{tabular}

Fuente: elaboración personal.

Tabla 6. Codificación por pregunta de las representaciones mentales en torno a la mujer

\begin{tabular}{|c|c|c|c|c|c|c|}
\hline 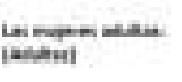 & 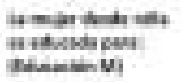 & 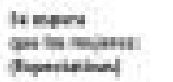 & 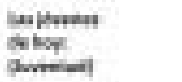 & 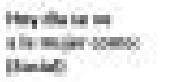 & in mop ant & 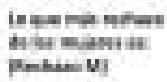 \\
\hline Itatulat & I Howh & 1an & If Hrither & 1 fandi & 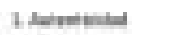 & 1 Hmin \\
\hline 1. Howarn & I Heith & $I=-t+r e s$ & 1 tonthos & ithedra & I whe & 3 sempara \\
\hline Inde & LEded & Ening & ctrindu & 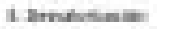 & $10+1$ & I child: \\
\hline inta & Anth & Ecuphintal & it Ehithem & 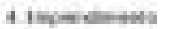 & a coldt & A to gais \\
\hline i pwats & Etw & kwarest & Inesta & inged & 1. now & hollowh \\
\hline itothes & thane- & t the & A Cimpang & +1.2 & A ints: & Sthwernios \\
\hline IIfni-n & Aismite & Fiter bust & Timbe & It. & Allowes & ingut \\
\hline if tomin & inters & A Hones & $1+$ & in tum & int & Iramian \\
\hline If frobin & Prat & Mith & mpitsital & a mont & if Antly & 9 पтени \\
\hline ro.ten & II Fintur & IIfann & 19 Fate & merry. & Finder & Thitrone \\
\hline If Rrmedit & IIt thyt & II Penateq & If hrows & 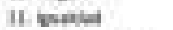 & $11=$ & if ontainth \\
\hline If $=5$ & F $\mathrm{n}=4 \mathrm{H}$ & IFraty & Mkente & 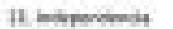 & F natili & I brom \\
\hline II tailu & intwhethat & Hille & intust & in thith & at byphith & 7. Urhare \\
\hline Af Exth & lis uthe & iffirts & 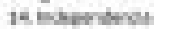 & Hitsd & H uthe & I \& silit \\
\hline il mandil & High & Atorsty & Iensin & Ih ketil & Il ikent & ditak \\
\hline Kenteri. & if burrest & iflay & IE rea & Miben & Ding & Wintele \\
\hline Ir tepiat & If rowhy & II tyalin & ir impiesithal & Tr.Melas= & If under & It rewan \\
\hline nimping & LPringeria & Intwatiou & nitur & numa & 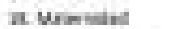 & A rithan \\
\hline notsed & lipwein & is Linui & II ithid & thesures & in subin & it the \\
\hline Muth & natorated & Wilare & wenitura: & buter & Dheredi & $x y=$ \\
\hline II mike & h spoten & hent.m & 1. month & inheren & it them & It hevert \\
\hline Hu. & Btorin & Ftuth & Mond & inesh & II Expintul & in \\
\hline ii $=1$ & iavilet & nithond & interes & it howply & if toty & \\
\hline If hmat & & Af theminda & 14 the & if athen. & If her & \\
\hline nitheth & & Artiminis & illomis & Romiden & $n=-$ & \\
\hline 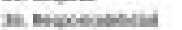 & & in & in whing & & H thingith & \\
\hline If htoth & & Heners & Fithowety & & it nte & \\
\hline 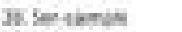 & & 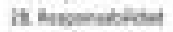 & & & 4 esurn & \\
\hline Pitheun & & 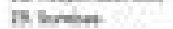 & & & & \\
\hline blonstan & & us bus & & & & \\
\hline ulerent & & & & & & \\
\hline
\end{tabular}

Fuente: elaboración personal. 
Tabla 7. Codificación por pregunta de las representaciones mentales en torno al hombre

\begin{tabular}{|c|c|c|c|}
\hline $\begin{array}{l}\text { El hombre se encarga de: } \\
\text { (Deber) }\end{array}$ & $\begin{array}{l}\text { El hombre desde niflo es educado: } \\
\text { [fducatión H] }\end{array}$ & $\begin{array}{l}\text { Se espera que los hombres: } \\
\text { [Expectativas H] }\end{array}$ & $\begin{array}{l}\text { Lo que mis rechano de los hombres: } \\
\text { [Rechazo H] }\end{array}$ \\
\hline $\begin{array}{l}\text { 1. Apoyo } \\
\text { 2. Criar }\end{array}$ & $\begin{array}{l}\text { 1. Amabilidad } \\
\text { 2. Bondad }\end{array}$ & $\begin{array}{l}\text { 1. Capaces } \\
\text { 2. Comprensión }\end{array}$ & $\begin{array}{l}\text { 1. Agresividad } \\
\text { 2. Arroganda }\end{array}$ \\
\hline $\begin{array}{l}\text { 3. Dificultad } \\
\text { 4. Dinero }\end{array}$ & $\begin{array}{l}\text { 3. Control } \\
\text { 4. Convivencia }\end{array}$ & $\begin{array}{l}\text { 3. Criar } \\
\text { 4. Dinero }\end{array}$ & $\begin{array}{l}\text { 3. Cobardia } \\
\text { 4. Desiguaklad }\end{array}$ \\
\hline 5. Estereotipo & 5. Criar & 5. Equidad & 5. Discriminación \\
\hline 6. Exito & 6. Desigualdad & 6. Esterectipes & 6. Ereradecimiento \\
\hline 7. Famila & 7. Dinero & 7. Exito & 7. Equidad \\
\hline 8. Felicidad & 8. Eeblatras & 8. Familia & 8. Estereotipos \\
\hline 9. Hogar & 9. Estereotipos & 9. Fortaleza & 9. Ignorancia \\
\hline 10. Igualdad & 10. Estudios & 10. Hoear & 10. Indiferencis \\
\hline 11. Independerncia & 11. Exito & 11. Honestidad & 11. Intolerancia \\
\hline 12. Iniclativa & 12. Felicidad & 12. lyualdad & 12, Iresponsables \\
\hline 13. Laboral & 13. Hogar & 13. Independencis & 13. Machismo \\
\hline 14. Uider & 14. leualdad & 14. Integridad & 14. Na contestó \\
\hline 15. No cantestó & 15. Independencia & 15. Laboral & 15. Orgullo \\
\hline 16. Objeto & 16. Integridad & 16. Libertad & 16. Prejuicios \\
\hline 17. Proteger & 17. Laboral & 17. Lideres & 17. Prepotencia \\
\hline 18. Proveedor & 18. Lider & 18. Machismo & 18. Presunción \\
\hline 19. Puealicaxión & 19. Machismo & 19. Madurez & 19. Respeto \\
\hline 20. Respeto & 20. No contestó & 20. Misandria & $20 . \operatorname{sen} 0$ \\
\hline 21. Responsablidad & 21. Obediencia & 21. No contestó & 21. Superiaridad \\
\hline 22. Puders & 22. Paternidsd & 22. Patemidad & 22. Violencis \\
\hline \multirow[t]{10}{*}{ 23. Superacibn } & 23. Proteceidn & 23. Perseverancia & \\
\hline & 24. Proveedor & 24. Protección & \\
\hline & 25. Rezlización & 25. Proveeder & \\
\hline & 26. Respeto & 26. Realización & \\
\hline & 27. Responsabilidad & 27. Respeto & \\
\hline & 28. Rudeza & 28. Responsabilidad & \\
\hline & 29. 50 ejemplo & 29. Rudeza & \\
\hline & 30. Superación & 30. Superación & \\
\hline & 31. Valiente & 31. Trascender & \\
\hline & & 32. Valentes & \\
\hline
\end{tabular}

Fuente: elaboración personal. 


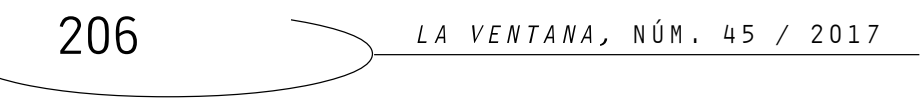

Tabla 8. Codificación por pregunta de las representaciones vinculadas a la identidad

\section{Los retos que tengo:}

(Metas personales)

1. Ambición

2. Bondad

3. Desigualdad

4. Desorganización

5. Dificultad

6. Diversión

7. Egoísmo

8. Esfuerzo

9. Espiritual

10. Estereotipos

11. Estudios

12. Éxito

13. Fortaleza

14. Independiente

15. Integridad

16. Laboral

17. No contestó

18. Perseverancia

19. Respeto

20. Riqueza

21. Social

22. Superación

23. Valiente
Lo que más aprecio de ser hombre/mujer:

(Autoestima)

1. Amabilidad

2. Amor

3. Aprecio

4. Autenticidad

5. Autoestima

6. Bondad

7. Conformismo

8. Convivencia

9. Desvalorización

10. Educación

11. Ególatra

12. Engradecimiento

13. Equidad

14. Ética

15. Éxito

16. Fortaleza

17. Identidad

18. Igualdad

19. Independencia

20. Libertad

21. Machismo

22. Maternidad

23. No contestó

24. Oportunidades

25. Pensamiento

26. Prejuicios

27. Respeto

28. Responsabilidad

29. Sabiduría

30. Seguridad

31. Sencillez

32. Son ejemplo

33. Superación

34. Visión
Me preocupa que se me considere:

(Preocupación)

1. Capaces

2. Conformismo

3. Débil

4. Desvalorización

5. Equidad

6. Estereotipos

7. Fracaso

8. Fuerte

9. Hogar

10. Homofóbico

11. Igualdad

12. Imponente

13. Incapacidad

14. Inferioridad

15. Inmadurez

16. Inseguridad

17. Irresponsabilidad

18. Laboral

19. Machismo

20. Maldad

21. No contestó

22. Objeto

23. Originalidad

24. Respeto

25. Sumisa

Fuente: elaboración personal. 
Figura 3. Mapa conceptual de las representaciones mentales de la equidad de género

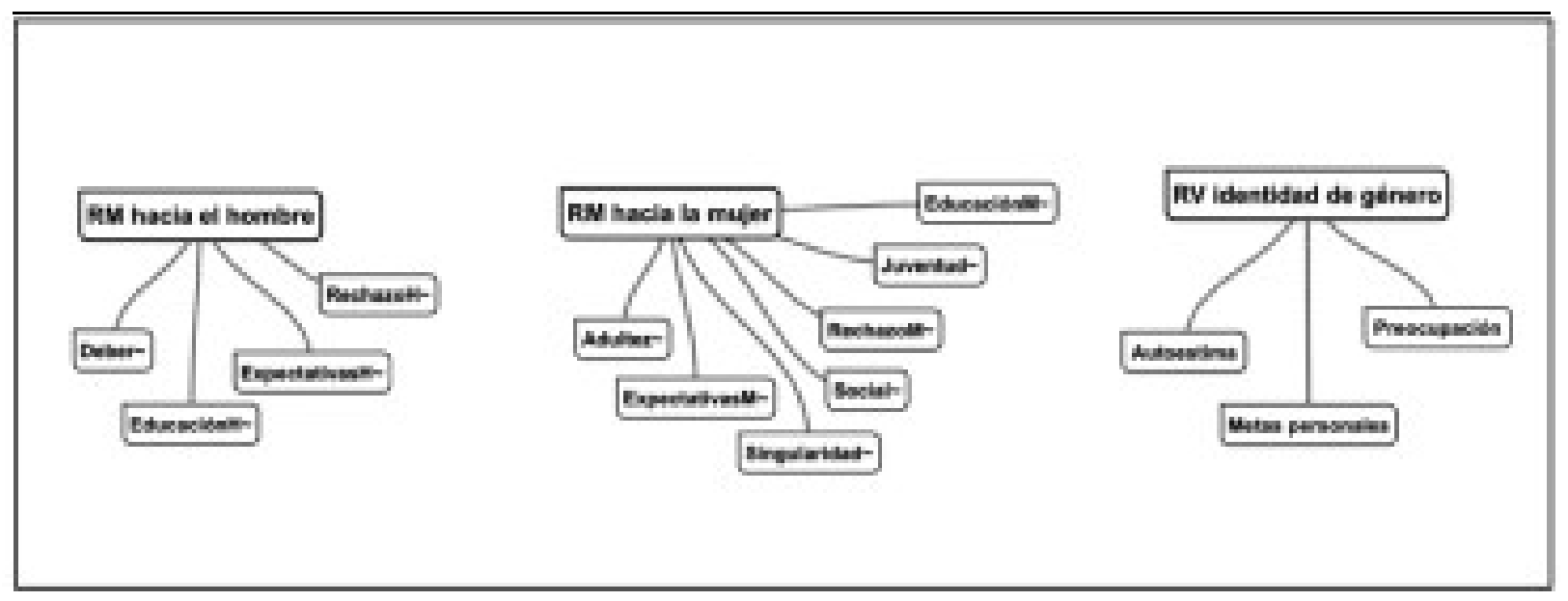

Fuente: elaboración personal.

\section{A manera de conclusión}

De los 131 códigos generados se encontró que los que tuvieron mayor iteración fueron, en el caso de las representaciones mentales en torno a la mujer, el ligado a la maternidad y, para el hombre, el ligado al ser proveedor. Este dato contrasta fuertemente con un discurso manifiesto que defiende la igualdad, en el que está presente también el deseo de superación personal, lo que arroja datos de una representación social muy arraigada respecto a lo que se espera de la mujer y del hombre.

Si bien hubo respuestas con connotaciones de misandria, estereotipos o desvalorización de un género para con otro, no fue la tendencia más fuerte. Destaca el hecho de una alta incidencia de 
"no contestó" lo cual habla de la necesidad de insistir en la discusión de estos temas que permitan al estudiante familiarizarse con una cultura de valores que promueva la equidad de género y la justicia social.

La equidad de género está muy enlazada a la justicia social y a la formación ciudadana en tanto provee de elementos que permiten asignar lo que a cada uno le corresponde en condiciones que reconocen los grupos minoritarios y que históricamente han sido vulnerados. El desafío para la institución educativa es grande ya que implica un trabajo paulatino de ruptura con estereotipos y representaciones sociales y culturales, ruptura que puede lograrse a través de la discusión y acercamiento a estos temas, de manera que los nuevos conceptos de equidad se vayan familiarizando e introyectando en las representaciones mentales de mujeres y hombres, se manifiesten en la cultura organizacional cotidiana de la escuela y puedan de a poco y cada vez más acrecentar las posibilidades de transformar la realidad que se vive hacia sociedades con mayor justicia.

Como resultado de esta investigación se ha implementado un sistema computarizado que pretende seguir recaudando de manera anónima la información respecto al constructo de equidad de género mediante el instrumento de frases incompletas, lo que permitirá profundizar en la comprensión de este fenómeno y generar espacios de reflexión apoyados en las nuevas tecnologías tan socorridas por la comunidad estudiantil. Se hace pertinente insistir en la discusión de estos temas que permitan al estudiante familiarizarse con una cultura de valores que promueva la equidad de género y la justicia 
social, generando los espacios que aborden estas problemáticas desde varios frentes y con ello se pueda transformar la realidad hacia sociedades más éticas que respondan a la formación ciudadana.

\section{Bibliografía}

Buttrago, María del Pilar; Cabrera, Karol y Guevara, Mónica (2009, diciembre). Las representaciones sociales de género y castigo y su incidencia en la corrección de los hijos. Educación y educadores. Recuperado de http://educacionyeducadores.unisabana.edu.co/index.php/ eye/article/view/1531/1837

Bernstein, Basil (1993). La estructura del discurso pedagógico. Madrid: Morata.

Bourdieu, Pierre (2008). Capital cultural, escuela y espacio social. (Trad. I. Jiménez). Madrid: Siglo XXI.

Camberos, María Teresa (2011). Empoderamiento femenino y políticas públicas, una perspectiva desde las representaciones sociales de género. Entramado. Recuperado de http://www.redalyc.org/articulo. oa? $\mathrm{id}=265422684003>$ ISSN 1900-3803

DeWEY, John (1998). Democracia y educación. España: Morata.

GIDDENS, Anthony (2000). Un mundo desbocado. Los efectos de la globalización en nuestros días. España: Taurus.

Guevara, Gilberto (1998). Democracia y educación. Cuadernos de divulgación de una cultura democrática. México, IFE. Consultado en línea en http://www.ine.mx/documentos/DECEYEC/democracia _y_educacion.htm 
GonZÁLEZ, Teresa (2010). Democracia y formación ciudadana. México: IFE. Recuperado de: http:/www.ife.org.mx/docs/IFEv2/ DECEYEC/ EducacionCivica/CuadernosDivulgacion/CuadernosDivulgacionpdfs/CUAD_28.pdf

Habermas, Jürgen (2007). Conocimiento e interés. México: Fondo de Cultura Económica.

LATAPí, Pablo (1993). Reflexiones sobre la justicia en la educación. Revista Latinoamericana de Estudios Educativos, Vol. XXIII, Núm. 2, pp. 9-41.

MATERÁN, Angie (2008). Las representaciones sociales: un referente teórico para la investigación educativa. Geoenseñanza. Recuperado de: http://www.redalyc.org/pdf/360/36021230010.pdf

MorA, Martín (2002). La teoría de las representaciones sociales de Serge Moscovici. Athenea Digital, Recuperado de http://www.raco.cat/ index.php/Athenea/article/viewFile/34106/33945

Organización de las Naciones Unidas para la Alimentación y la Agricultura (2011). Por qué género. Recuperado de: http://www.fao.org/gender/ gender-home/gender-why/por-que-el-genero/es/

Proigualdad: Centro de Estudios para el Adelanto de la Mujeres y la Equidad de Género (2013). Informe referido a políticas públicas y presupuesto federal para las mujeres y la igualdad de género en México, en el marco de las obligaciones del estado mexicano establecidas en la CEDAW. México: Cámara de Diputados.

Serret, Estela; Alfie, Miriam y García, María (1991, enero-abril). El problema de la desigualdad sexual. Más allá del feminismo. Sociológica. Recuperado de http://www.revistasociologica.com.mx/pdf/1514.pdf 